\title{
COnE)-(OES
}

CIÊNCIA E TECNOLOGIA

\section{PADRÕES DE COLORAÇÃO DE Ischnochiton striolatus (GRAY, 1828) (MOLLUSCA: POLYPLACOPHORA) EM CAMOCIM, CEARÁ}

\author{
Davi José Araújo de lima, Karina Aparecida Araújo Dutra, José Breno do Carmo Souza,
} RAFAela CAMARgo Maia

Instituto Federal de Educação, Ciência e Tecnologia do Ceará - IFCE <davilimaifce@yahoo.com>.<araujokarina807@gmail.com>.<breno1046.bc@gmail.com>. $<$ rafaelamaia@ifce.edu.br>

DOI: $10.21439 /$ conexoes.v15i0.2102

\begin{abstract}
Resumo. A Classe Polyplacophora é constituída por organismos com formato ovoide e caracterizados por apresentarem oito placas sobrepostas na região dorsal. O presente estudou analisou os padrões de coloração existentes na espécie Ischnochiton striolatus Gray (1828) realizando uma correlação dos padrões de cor com o tamanho dos organismos. A coleta ocorreu em três pontos ao longo da faixa de praia estuarina de Camocim - CE. Foi demarcado três quadrantes aleatórios com área de $25 \mathrm{~m}$ cada. Os poliplacóforos presentes dentro destes quadrantes foram coletados por meio do método de busca ativa, durante o tempo de 10 minutos com o esforço amostral de quatro observadores, sendo estes capturados e armazenados ainda vivos em sacos plásticos devidamente etiquetados. Os animais apresentaram quinze padrões diferente de polimorfismo de cor. A classe de tamanho mostrou abundância e riqueza de cor em organismos de tamanho médio. O padrão Bege destacou-se como mais abundante entre os indivíduos, e os padrões Caramelo e Rose com maior distribuição entre as categorias de tamanhos. O presente trabalho é considerado o primeiro a realizar possíveis relações entre polimorfismo de cor com o tamanho dos quítons. A espécie I. striolatus apresentou para tamanhos médios, maior frequência de riqueza cromática e abundância de indivíduos.
\end{abstract}

Palavras-chaves: Padrões de cor. Polimorfismo de cor. Quítons. Regiões costeiras.

\section{COLOR PATTERNS OF Ischnochiton striolatus (GRAY, 1828) (MOLLUSCA: POLYPLACOPHORA) IN CAMOCIM, CEARÁ}

\begin{abstract}
The Polyplacophora class consists of ovoid shaped organisms and characterized by having eight overlapping plaques in the dorsal region. The present study analyzed the color patterns existing in the species Ischnochiton striolatus Gray (1828) performing a correlation of the color patterns with the size of the organisms. The specimens were collected at three points along the estuarine beach strip of Camocim - CE. Three random quadrants with an area of $25 \mathrm{~m}$ each were demarcated. The polyplacophores present within these quadrants were collected using the active search method, over a during a 10-minute period with the sampling effort of four observers, which were captured and stored alive in properly labeled plastic bags. The animals showed fifteen different patterns of color polymorphism. The size class showed abundance and color richness in medium sized organisms. The Beige pattern stood out as the most abundant among individuals and the Caramel and Rose patterns with greater distribution among the size categories. The present work considered the first to make possible relationships between color polymorphism and the size of the chitons. The species I. striolatus presented, for medium sizes, a higher frequency of chromatic richness and abundance of individuals.
\end{abstract}

Keywords: Color patterns. Color polymorphism. Chitons. Coastal regions.

\section{INTRODUÇÃO}

A Classe Polyplacophora é constituída por organismos achatados ventralmente, caracterizados pela presença de oito placas na região dorsal (OKUSU et al. 2003). São descritos como animais exclusivamente ma- rinhos, podendo ser encontrados principalmente associados a substratos rochosos entre marés, havendo registros de algumas espécies em águas profundas e estuarinas temperadas (GIBERTO et al., 2004, ARIAS; ANADóN, 2013).

O polimorfismo de cor é comum em grande parte 
dos organismos bentônicos presentes em regiões costeira e tem como característica a presença de dois ou mais morfotipos de cores dentro de uma população determinada e geneticamente segregado no ambiente (GRAY; MCKINNON, 2007). A seleção natural atua sobre o polimorfismo das cores por meio da ação de predadores visuais (BOND; KAMIL, 1998), como aves que se alimentam de maneira seletiva desses animais polimórficos (JORMALAINEN; MERILAITA; TUOMI, 1995).

Em moluscos, como alguns gastrópodes, são comuns características de polimorfismos de cor em sua fisionomia, estabelecendo-se em regiões costeiras sob superfícies de rochosa onde os mesmos utilizam padrões variáveis de coloração da concha e outras estruturas corporais para proteção seu próprio ambiente (BLANCO; TAMAYO; SCATENA, 2014). O polimorfismo de cor em alguns destes indivíduos está fortemente relacionado com hábito alimentar e temperatura do ambiente (UNDERWOOD; CREESE, 1976; NYBAKKEN; BERTNESS, 2005).

Ischnochiton striolatus Gray (1828), habitante de zonas entre-marés de substrato consolidado (OSMAN, 1977; SMITH; OTWAY, 1997), possui valvas de aspecto estriado, sendo que sua valva cefálica possui formato semi circular e inclinação na região frontal reta; já as valvas intermediárias são amplas de forma retangular distribuídas pela região dorsal sobreposta umas as outras e por fim, a valva anal, possui largura maior que a valva cefálica com a margem frontal convexa, com um mucro central e inclinação posterior côncava (KAAS; BELLE, 1990).

Esta espécie apresenta polimorfismo de cor em sua região dorsal, as ações mecânicas das ondas, ocasiona o movimento das rochas onde esses animais estão, os expondo a predadores visuais, um fator que pode contribuir para a existência do polimorfismo, isso porque participam da cadeia alimentar servido de alimento para consumidores secundários, e consumindo alimentos primários como algas, (SOUSA, 1980).

Nesse contexto, o trabalho tem por objetivo geral analisar a ocorrência dos padrões de coloração da espécie I. striolatus coletadas em Camocim - CE. Especificamente, 1) Analisar os padrões da variância de cor dos organismos durante o período de amostragem; 2) Realizar a correlação dos padrões de cor com o tamanho dos quítons amostrados.

\section{METODOLOGIA}

\section{1 Área de Estudo}

O presente estudo foi realizado em uma faixa de praia estuarina rochosa sob influência do estuário do Rio Coreaú, no município de Camocim, Ceará (253'59" S 4050'47” W) (Figura11A). O município está localizado no litoral oeste do estado há $379 \mathrm{~km}$ da capital Fortaleza. A sua área de extensão territorial é de 1123,94 $\mathrm{km}^{2}$. A região é caracterizada por possuir o clima tropical semiárido brando (IPECE, 2012) com pluviosidade média anual no ano de 2017 foi de $92,250 \mathrm{~mm}$ respectivamente (FUNCEME, 2019).

A região possui uma faixa de praias arenosa de características morfodinâmicas dissipativas que realizam associações às planícies fluvio-marinha com presença de uma extensa área de manguezal (MORAIS et al. 2006). Os aspectos fisionômicos da região costeira presentes incluem recifes de arenito formado por grandes e pequenas rochas soltas na sua faixa de praia, assim como dunas e falésias regido por um regime de marés semi-diurno (Franklin Jr, 2005).

\subsection{Procedimento em Campo}

A coleta de quítons foi realizada nos meses de março, maio e julho de 2017, em períodos de maré baixa de sizígia de acordo com a tábua de marés obtida junto a Diretoria de Hidrografia e Navegação da Marinha Brasileira (DHN, 2017,2018). Foram selecionados três pontos, $\mathrm{P} 1\left(02^{\circ} 53.822^{\prime} \mathrm{S} 040^{\circ} 50.445^{\prime} \mathrm{W}\right)$; P2, (02 ${ }^{\circ} 53.143^{\prime}$ $\left.\mathrm{S} 040^{\circ} 50.742^{\prime} \mathrm{W}\right)$ e $\mathrm{P} 3\left(02^{\circ} 52.125^{\prime} \mathrm{S} 040^{\circ} 51.425^{\prime} \mathrm{W}\right)$ (Figura 1B).

Em cada ponto, foram demarcados, com auxílio de uma trena, três quadrantes aleatórios com área de $25 \mathrm{~m}$ cada. Os poliplacóforos presentes dentro destes quadrantes foram coletados por meio do método de busca ativa, durante o tempo de 10 minutos com o esforço amostral de 04 (quatro) observadores. Os indivíduos capturados foram armazenados ainda vivos em sacos plásticos devidamente etiquetados.

\subsection{Procedimento em Laboratório}

Após captura dos espécimes, os mesmos foram transportados ao Laboratório de Ecologia de Manguezais (ECOMANGUE) do IFCE Campus Acaraú, onde foram fixados e armazenados em álcool 70\%. Em seguida, os organismos foram fotografados na porção dorsal com o auxílio de uma câmera com resolução $4128 \times 3096$ pixels e depois medidos o comprimento com auxílio de uma régua.

Para a padronização do fundo de imagem foi utilizado um fundo branco e uma escala de medida. A 
A

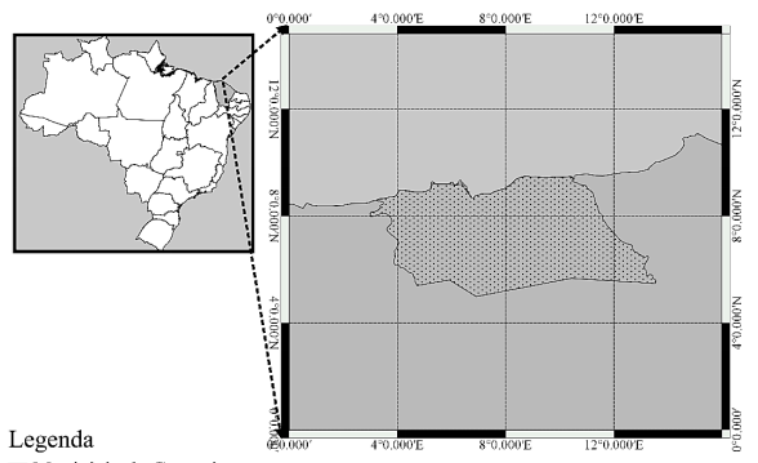

Município de Camocim $\square$ Estado do Ceará

classificação dos indivíduos de I. striolatus em morfotipos foi realizada de acordo com os seus padrões de coloração, sendo adotados para a classificação dos organismos as cores que apresentavam predominância nos indivíduos.

\subsection{Análise de dados}

Para análise de dados foram feitos histogramas de frequência para analisar as classes de tamanhos dos organismos com os padrões de cor localizado. Para isso foi utilizado a fórmula de Sturges: $\mathrm{Vi}=\mathrm{A} / \mathrm{K}$, onde $\mathrm{Vi}=$ Intervalo de classes, $\mathrm{A}=$ Amplitude da variável (número máximo menos o número mínimo), $\mathrm{K}=$ número de classes, calculado pela fórmula: $1+3,32 \log n$, onde "n" representa o número total de indivíduos. Para o presente estudo o cálculo resultou em intervalos de classes de tamanho de $1,5 \mathrm{~mm}$. Dessa maneira foram determinadas 8 classes de tamanhos.

\section{RESULTADOS}

\subsection{Descrição dos padrões de cores}

Os organismos I. striolatus coletados constituíram 15 padrões diferentes de cores sendo eles: bege escuro (A1), caramelo (A2), rosé (A3), mesclado tipo 1 (B1), mesclado tipo 2 (B2), mesclado marrom (B3), marfim (C1), creme (C2), musgo (C3), marrom (D1), carmim (D2), verde(D3), verde claro (E1), musgo tipo 1 (E2) e musgo tipo 2 (E3) como apresentado na Figura 2

$\mathrm{O}$ primeiro padrão representado por $\mathrm{A} 1$ foi determinado a partir de organismos que apresentavam coloração bege com ou sem manchas. No padrão A2 se enquadraram os indivíduos de coloração amarela e que não apresentavam machas. Para estabelecer o padrão
B

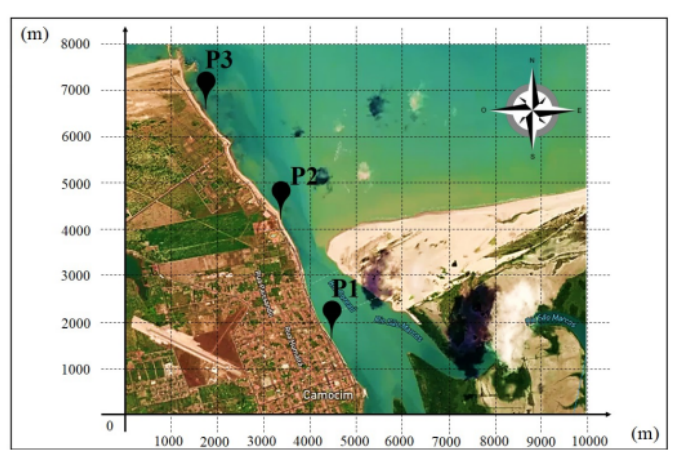

A3 foram agrupados os organismos que apresentavam coloração rosada apresentando ou não manchas na área jugal.

Os padrões B1, B2 e B3 foram determinados para organismos com mesclas de outras cores, que variavam de manchas castanha, verde escuras em todas as valvas ou não, sendo classificados respectivamente como mesclado tipo1, mesclado tipo 2 e mesclado marrom. No padrão Marfim representado por $\mathrm{C} 1$, foram agrupados os organismos que apresentaram a cor branca próximo ao tom marfim, com ou sem machas claras. Na categoria Creme representado pelo padrão $\mathrm{C} 2$ foram enquadrados os organismos que apresentavam aspecto de coloração branca com manchas nas laterais e no centro do animal semelhante a cor creme.

Para o padrão D1 foram classificados os organismos de cor marrom que não apresentavam manchas. $\mathrm{O}$ padrão Carmim representado por D2, foi determinado para os organismos de coloração vermelha com faixa branca na parte central das valvas. Os organismos que apresentavam coloração verde com tonalidades escurecidas foram agrupados no padrão Verde representados por D3, e os animais com cores verdes sem tonalidades escuras classificadas em Verde claro sendo representados por E1.

A cor Musgo foi determinada para os padrões C3, E2 e E3. No padrão C3 os organismos foram classificados como Musgo por apresentarem coloração esverdeada em um tom intermediário ao verde escuro, já o padrão E2 foram agrupados os organismos de cor Musgo em tons claros sendo classificado os como Musgo tipo 1. Para o padrão E3 os indivíduos com tonalidade mais escura e com manchas pretas foram classificados como Musgo tipo 2. 
A1

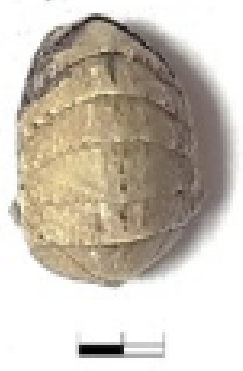

B2

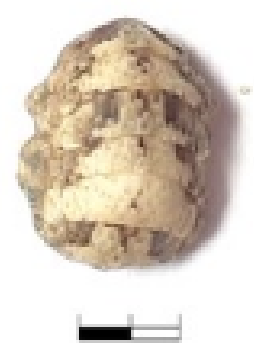

C3

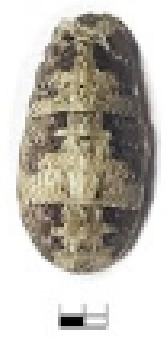

E1

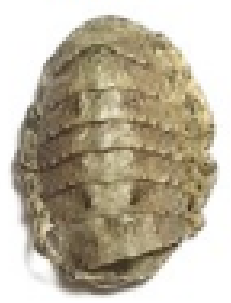

A2

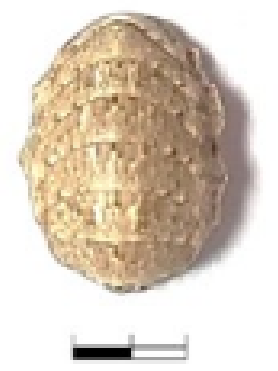

B3

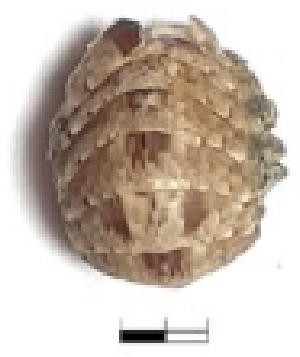

D1

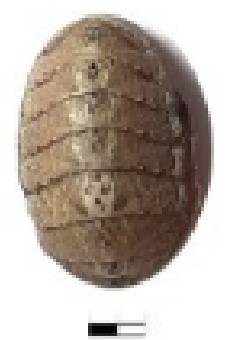

E2

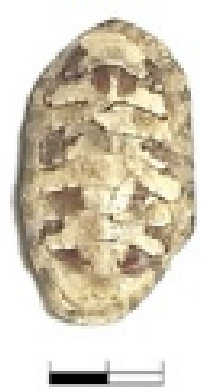

A3

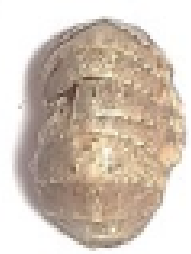

1

C1

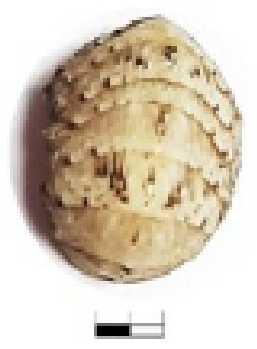

D2

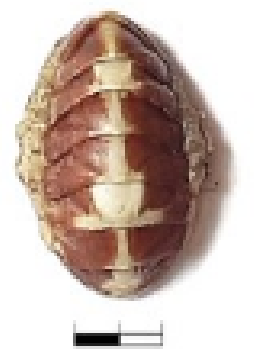

E3

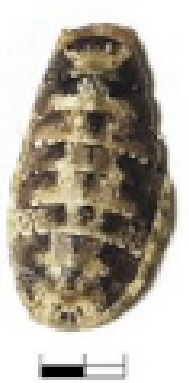

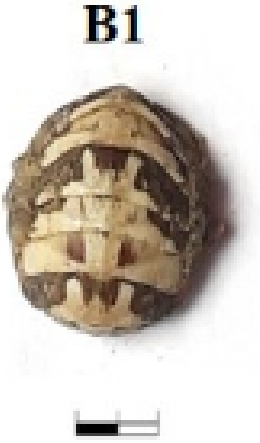

C2

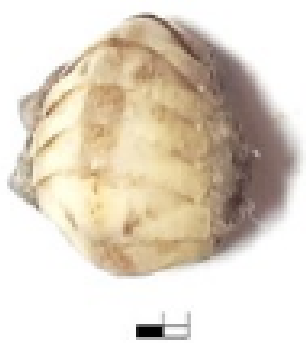

D3

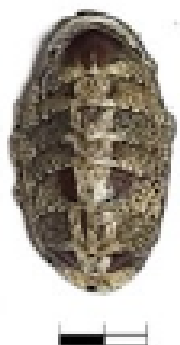

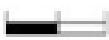




\subsection{Frequência de Tamanho e Padrões de Cor}

Foram coletados 146 indivíduos da espécie I. striolatus, com tamanhos variando de 4,5 a $16,5 \mathrm{~mm}$. A maior abundância de organismos foi observada entre 7,5 e 12 mm apresentando $77 \%$ de indivíduos (Figura 3 ).

Figura 3: Frequência de indivíduos por intervalo de classe (mm).

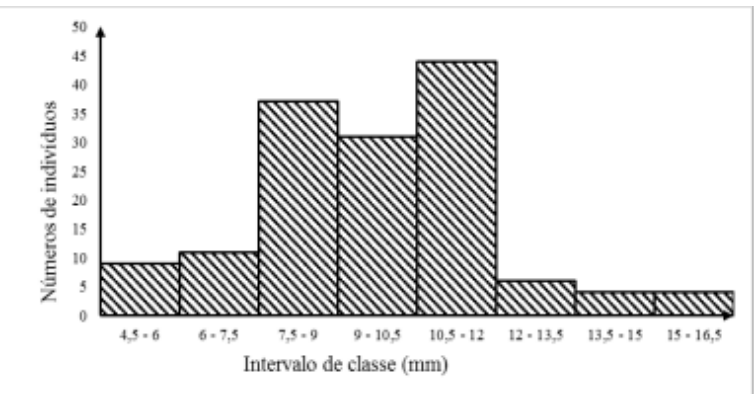

Em todas as classes de tamanho foram observadas mais de um padrão de cor, variando de quatro a oito nas classes entre 4,5 e 10,5 mm. Já nas classes 12 e 16,5 $\mathrm{mm}$ foram presenciado entre duas e dez cores (Figura 4).

Figura 4: Frequência do número de padrões de cores entre cada classe de tamanho dos indivíduos.

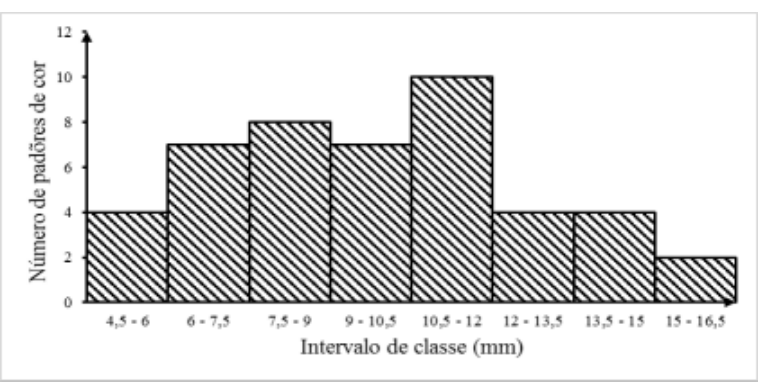

O padrão A1 foi o considerado a cor mais comum neste estudo. Entretanto, os padrões A3 e A2 tiveram maior distribuição entre as classes de tamanho (Figura 5] Já na classe de tamanhos de 15 a $16 \mathrm{~mm}$ foi observado a presença de apenas dois padrões de cor A3 e E1. $\mathrm{O}$ padrão $\mathrm{A} 1$, foi o abundante entre os indivíduos, e os padrões A2 e A3 com maior distribuição entre as classes de categorias de tamanhos.

A espécies I. striolatus é presente quase todas as regiões costeiras do Brasil inclusive no estado do Ceará (SIMONE; JARDIM, 2009, ROCHA-BARREIRA et al. 2017). Os padrões de cores corroboram com a literatura taxonômica destes organismos podendo apresentar mais de um padrão de cor (KAAS; BELLE, 1990 RODRIGUES; ABSALãO, 2005).
Figura 5: Distribuição dos padrões de cor de I. striolatus entre as classes de tamanho.

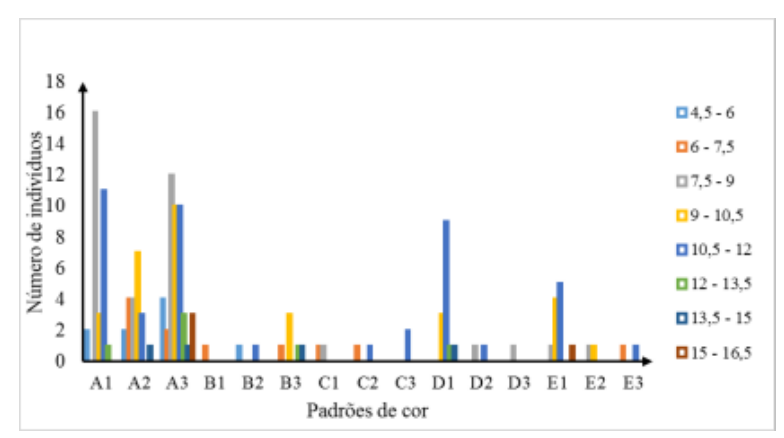

$\mathrm{Na}$ literatura existente, não existem trabalhos que façam relações de tamanho e polimorfismo de cor na Classe Polyplacophora. Rodrigues e Absalão (2005) avaliaram as variações de cor em I. striolatus e mostraram uma relação de riqueza de cor com tamanho médio das rochas do habitat. O trabalho de Mendonça et al. (2015) também relacionou espécies de quítons com o substrato e reportou interações significativas da riqueza de cor dos organismos com ambiente rochosos com predominância de algas. Entretanto, não retratam possíveis relações entre variabilidade de cor com o tamanho dos organismos.

O resultado da frequência das classes de tamanho observado neste estudo mostrou que os organismos de tamanho médio apresentam maior riqueza cromática, como também maior abundância de indivíduos. Portanto, o tamanho corporal médio destes indivíduos pode ter forte relação com a variação da salinidade presente no ambiente estuarino, uma vez que estas variações podem impedir que a comunidade de quítons possa se estabelecer nestes ambientes por muito tempo. Esta hipótese corrobora com trabalho de Eernisse, Clark e Draeger (1994) e Schwabe (2010) na qual relatam que os quítons possuem maior preferência por ambientes marinhos.

Além disso, a seleção natural pode atuar no polimorfismo de cor por meio da ação de predadores visuais, o que também pode interferir no tamanho médio dos indivíduos encontrados como é relatado nos trabalhos de Cain e Sheppard (1950) e Gardner (1995).

Dos organismos coletados, $77 \%$ pertenciam entre as classes de tamanho 7,5 e $12 \mathrm{~mm}$. A Abundância de organismos e o tamanho pode estar relacionado com a disponibilidade de nutrientes no ambiente amostrado, como presença de macroalgas, pois, segundo (CORREIA; COELHO; SOVIERZOSKI, 2015), esses moluscos se alimentam de diatomáceas e algas verdes presentes no biofilme comumente encontrado nessas algas. 
PADRÕES DE COLORAÇÃO DE Ischnochiton striolatus (GRAY, 1828) (MOLLUSCA: POLYPLACOPHORA) EM CAMOCIM, CEARÁ

Um estudo realizado com o gastrópode Littoraria filosa (G. B. Sowerby I, 1832) mostrou que a espécie apresenta característica de polimorfismo na coloração de suas conchas, porém as análises de tamanho, não apresentaram resultados significativos com a predominância de coloração (REID, 1987).

O polimorfismo de cor nos moluscos geralmente está associado ao mecanismo de predação podendo levar algumas espécies adotarem um padrão de cor associado ao ambiente como forma de proteção. Em um estudo feito por Blanco, Tamayo e Scatena (2014) mostrou que o gstrópode Neritina virginea (Linnaeus, 1758) muda o padrão de coloração de sua concha após estas serem danificadas pela ação de predadores.

Os hábitos alimentares provenientes das regiões costeiras também podem influenciar na coloração de alguns moluscos podendo realizar uma possível relação com tamanho. (UNDERWOOD; CREESE, 1976 mostrou que a pigmentação da concha de gastrópodes Austrocochlea constricta (Lamarck, 1822) depende da dieta do mesmo, onde as células do manto do animal possuem forte afinidade com a clorofila presente nas algas na qual se alimentavam.

\section{CONSIDERAÇÕES FINAIS}

O presente trabalho é considerado o primeiro a realizar possíveis relações entre polimorfismo de cor com o tamanho dos quítons. A espécie I. striolatus apresentou para tamanhos médios, maior riqueza cromática e abundância de indivíduos. No entanto, novos estudos com demanda de tempo maior de amostragem são necessários para melhor compressão do comportamento cromático destes indivíduos, como também relacionar o polimorfismo de cor destes animais outras variáveis ambientais, como tipo de substratos e salinidade.

\section{AGRADECIMENTOS}

Os autores agradecem ao Instituto Federal de Educação, Ciência e Tecnologia do Ceará (IFCE) - Campus Acaraú pelo deslocamento para a realização deste trabalho. Ao Laboratório de Ecologia de Manguezais (ECOMANGUE) pelo apoio à pesquisa e ao Prof. Dr. Jaime Jardim pelo suporte taxonômico.

\section{REFERÊNCIAS}

ARIAS, A.; ANADóN, N. N. tonicia atrata and chiton cumingsii (polyplacophora: Chitonidae): First records in european waters. Zootaxa, v. 3626, n. 4, p. 593-596, Mar. 2013. Disponível em: <https://www.mapress. com/zt/article/view/zootaxa.3626.4.14>
BLANCO, J. F.; TAMAYO, S.; SCATENA, F. N.

Variación fenotípica de la concha en neritinidae (gastropoda: Neritimorpha) en ríos de puerto rico. Revista Biologia Tropical, v. 62, n. 1, p. 53-68, 2014.

BOND, A.; KAMIL, A. C. Apostatic selection by blue jays produces balanced polymorphismin virtual prey.

NATURE, v. 395, n. 1, p. 594-596, 1998.

CAIN, A. J.; SHEPPARD, P. M. Selection in the polymorphic land snail cepæa nemoralis. Heredit, v. 4, n. 1, p. 275-294, 1950.

CORREIA, M. D.; COELHO, C. A.; SOVIERZOSKI, H. H. Polyplacophora (mollusca) from reef ecosystems and associations with macroalgae on the coast of alagoas, northeastern brazil. Zoologia, v. 32, n. 4, p. 289-295, 2015.

\section{DHN. DIRETORIA DE HIDROGRAFIA E} NAVEGAÇÃO DA MARINHA BRASILEIRA. 2017. Tábua de maré Camocim CE. Disponível em: $<$ https://www.marinha.mil.br/dhn/>

EERNISSE, D. J.; CLARK, R. N.; DRAEGER, A. Polyplacophora. Microscopic Anatomy of Invertebrates, v. 5, n. 1, p. 352-354, 1994.

Franklin Jr, W. Levantamento da macrofauna bentônica de ambientes inconsolidados do estado do Ceará (faixa de entre-marés de praias arenosas). 2005. Programa: Zoneamento Ecológico e Econômico (ZEE) da Zona Costeira do Estado do Ceará. Governo do Ceará.

FUNCEME. Posto Camocim. Camocim: FUNDAÇÃO CEARENSE DE METEOROLOGIA E RECURSOS HÍDRICOS, 2019. Disponível em: <www.funceme.br/> Acesso em: 20 mar.

GARDNER, M. G. Relationship between shell-pattern frequency and microhabitat variation in the intertidal prosobranch clithon qualaniensis (lesson). Malacologia, v. 36, n. 1, p. 97-109, 1995.

GIBERTO, D. A.; BREMEC, C. S.; ACHA, E. M.; MIANZAN, H. Large-scale spatial patterns of benthic assemblages in the sw atlantic: the río de la plata estuary and adjacent shelf waters. Estuarine, Coastal and Shelf Science, v. 61, n. 1, p. 1-13, 2004.

GRAY, S. M.; MCKINNON, J. S. Linking color polymorphism maintenance and speciation. Trends in Ecology Evolution, v. 22, n. 1, p. 71-79, 2007. 
PADRÕES DE COLORAÇÃO DE Ischnochiton striolatus (GRAY, 1828) (MOLLUSCA: POLYPLACOPHORA) EM CAMOCIM, CEARÁ

JORMALAINEN, V.; MERILAITA, S.; TUOMI, J. Differential predation on sexes affects colour polymorphism of the isopod idotea baltica (pallas). Biological Journal of the Linnean Society, v. 55, n. 1, p. 45-68, 1995.

KAAS, P.; BELLE, R. A. V. Monograph of living chitons: (mollusca: Polyplacophora), suborder ischnochitonina: Ischnochitonidae: Ischnochitoninae (continued), additions to vols. 1, 2 y 3. E.J. Brill, Leiden, v. 4, n. 1, p. 298, 1990.

MENDONçA, V.; VINAGRE, C.; CABRAL, H.; SILVA, A. C. F. Habitat use of inter-tidal chitons - role of colour polymorphism. Marine Ecology, v. 36, n. 1, p. 1098-1106, 2015.

MORAIS, J. O.; FREIRE, G. S. S.; PINHEIRO, L. S.; SOUZA, M. J. N. D.; CARVALHO, A. M.; PESSOA, P. R. S.; OLIVEIRA, S. H. M. Caracterização fisiográfica e geoambiental da zona costeira do estado do ceará. In: MUEHE, D. (Ed.). Erosão e Progradação do Litoral Brasileiro. Rio de Janeiro: MMA (Ministério do Meio Ambiente), 2006. p. 132 154.

NYBAKKEN, J. W.; BERTNESS, M. B. Marine Biology: an ecological approach. 2005. 144-332 p.

OKUSU, A.; SCHWABE, E.; EERNISSE, D. J.; GIRIBET, G. Towards a phylogeny of chitons (mollusca, polyplacophora) based on combined analysis of five molecular loci. Organisms Diversity Evolution, v. 3, n. 1, p. 281-302, 2003.

OSMAN, R. W. The establishment and development of a marine epifaunal community. Ecological Monographs, v. 47, n. 1, p. 37-63, 1977.

REID, D. G. Natural selection for apostasy and crypsis acting on the shell colour polymorphism of a mangrove snail, littoraria fzlosa (sowerby) (gastropoda: Littorinidae). Biological Journal of the Linnean Society, v. 30, n. 1, p. 1-24, 1987.

ROCHA-BARREIRA, C. A.; BARROS, K. V. S.; MATTHEWS-CASCON, H.; QUEIROZ, L. R.; SILVA, A. F.; BARROSO, C. X. Advances in animal science and zoology. In: 1. ed. Máxico: Nova Science Publishers, 2017. v. 10, cap. Ecology of Mollusc Communities in Marine Environments: Central Region of the Semiarid Coast of Brazil, p. 39. ISBN 978-1-53612-024-0.

RODRIGUES, L. R. G.; ABSALãO, R. S. Shell colour polymorphism in the chiton ischnochiton striolatus (gray, 1828) (mollusca: Polyplacophora) and habitat heterogeneity. Biological Journal of the Linnean Society, v. 85, n. 1, p. 43-548, 2005.

SCHWABE, E. Illustrated summary of chiton terminology (mollusca, polyplacophora). Spixiana, v. 33, n. 2, p. 171-194, 2010.

SIMONE, L.; JARDIM, J. Classe polyplacophora. In: RIOS (Ed.). Sheashells of Brazil. 2. ed. Rio GRande: Evangraf, 2009.

SMITH, K. A.; OTWAY, N. M. Spatial and temporal patterns of abundance and effects of disturbance on under-boulder chitons. Molluscan Research, v. 18, n. 1, p. 43-57, 1997.

SOUSA, W. P. The responses of a community to disturbance: The importance of successional age and species' life histories. Source: Oecologia, v. 45, n. 1, p. 72-81, 1980.

UNDERWOOD, A. J.; CREESE, R. Observations on the biology of the trochid gastropod austrocochlea constrzcta (lamarck) (prosobranchia). ii. the effects of available food on shell-banding pattern. Journal of Experimental marine biology and ecology, v. 23, n. 1, p. 229-240, 1976. 